\title{
ARTICLE
}

\section{Analysis of radioinduced DNA damages using Monte Carlo calculations at nanometric scale for different irradiation configurations}

\author{
Carmen Villagrasa $^{\mathrm{a}, \mathrm{b}^{*}}$, Gaëtan Gruel ${ }^{\mathrm{a}}$, Joan-Françesc Barquinero ${ }^{\mathrm{a}}$, Pascale Voisin ${ }^{\mathrm{a}}$, Isabelle Clairand ${ }^{\mathrm{a}, \mathrm{b}}$ and \\ Jean-François Bottollier-Depois ${ }^{\mathrm{a}, \mathrm{b}}$ \\ ${ }^{a}$ Institute for Radiological Protection and Nuclear Safety, IRSN, BP-17, 92262-Fontenay-aux-Roses, France; ${ }^{b}$ the authors are \\ members of the Geant4-DNA collaboration
}

\begin{abstract}
The mean absorbed dose provides an adequate quantity with regards to the induced biological effects in many applications of ionizing radiations. However, in certain conditions, it does not give a description of the irradiation characteristics detailed enough that would allow predicting properly biological consequences. In particular, the absorbed dose can be misleading when the degree of heterogeneity in the energy deposited in sites exposed to the same radiation field is important compared to the target size. This is the case when investigating the number of radioinduced double strand breaks in the DNA content of a cell population. The study of the heterogeneity in the energy deposition in these nanometric volumes is now possible thanks to the use of Monte Carlo dedicated codes. Indeed, Monte Carlo codes have evolved to include electron transport to very low energy allowing a nanometric description of the energy deposited structure within living matter. In the present work, the Geant4-DNA Monte Carlo processes have been used to study the distribution of the specific energy deposited by photons, taking into account the size and shape of the DNA target of a cell nuclei population. The results of the simulations for a ${ }^{137} \mathrm{Cs}$ irradiation were compared, for the same macroscopic absorbed dose, to the $\gamma$-H2AX foci distributions obtained by an immunofluorescence technique that are related to the detection of DNA double strand breaks by the cell. The comparison of these simulated and experimental distribution variances appears as a possible method to link the irradiation parameters to their early biological consequences. In addition, the study of the evolution of the relative specific energy variance with the mean absorbed dose, in the case of ${ }^{137} \mathrm{Cs}$ irradiation obtained by Monte Carlo calculation, is also presented on this work. Finally, preliminary results on the calculated specific energy distributions obtained for ${ }^{137} \mathrm{Cs}$ and $20 \mathrm{keV} \mathrm{X}$-rays were compared in order to study the effect of the radiation quality.
\end{abstract}

Keywords: Monte Carlo; track structure; nanometric scale; heterogeneity of the energy deposition; $\gamma-\mathrm{H} 2 \mathrm{AX}$ foci distributions; DNA target

\section{Introduction}

Radioinduced DNA double strand breaks (DSB) are believed to be important early damages responsible for late effects as chromosomal aberrations or cell death. The topological and quantitative analysis of these damages is now possible using immunofluorescence techniques [1]. Indeed, when a DSB occurs, many molecules of histone $\mathrm{H} 2 \mathrm{AX}$ become phosphorilated $(\gamma-\mathrm{H} 2 \mathrm{AX})$ along megabase chromatin domains adjacent to the break as an early step of the DSB signaling and repair. $\gamma-\mathrm{H} 2 \mathrm{AX}$ histones can then be visualized as foci by immunofluorescence using phospho-specific antibodies.

Distributions of $\gamma-\mathrm{H} 2 \mathrm{AX}$ around a mean value can then be obtained from a large population of cell nuclei exposed to radiation at low doses [2]. Differences in the

*Corresponding author. Email: Carmen.villagrasa@irsn.fr mean value (given in terms of foci/cell/Gy) have been observed depending on the radiation quality [3]. Nevertheless, the variance of these distributions around the mean value has not yet been studied in detail.

In most of the experimental data obtained by this technique, the irradiation exposure is given in terms of the absorbed dose defined as the expected value of the energy imparted per mass at a point. The absorbed dose is an appropriate description of the energy deposited only if the number of events is large enough that the variation in energy deposited in individual targets of concern is small.

When studying the number of $\gamma-\mathrm{H} 2 \mathrm{AX}$ induced by ionizing radiation in a cell nuclei population, the target of concern is the DNA content. Due to the geometry and size of the DNA molecule, the degree of heterogeneity in the energy deposited within the target must be studied and taken into account. Monte Carlo codes can reproduce with the nanometric required precision the 
stochastic nature of physical interactions occurring between particles and matter. The purpose of the present work was to calculate by using the Geant 4 Monte Carlo code [4] the distribution of the specific energy within the target of interest, and study the evolution of this distribution with the absorbed dose, the target size and the radiation quality in order to highlight if the heterogeneity in the energy deposition at the DNA scale can be directly related to the variance in the number of early radioinduced foci.

\section{Materials and methods}

\subsection{Physical models used in the Monte Carlo calculation}

Energy deposited within a large population of cell nuclei for two photon radiation qualities $\left({ }^{137} \mathrm{Cs}\right.$ and 20 $\mathrm{keV}$ X-rays) has been calculated using the Monte Carlo code Geant4 [4] (versions 9.4 and 9.5 respectively). The primary particle transport was carried out taking into account the Compton scattering, photoelectric effect, pair production and Rayleigh scattering from the Livermore models implemented in the code. Nevertheless, for photon irradiations, secondary electrons are the main responsible for the energy deposition in the target. In particular, the nanometric precision on the energy track structure needed in this work was achieved using the Geant4-DNA processes [5] of the low energy package. These processes allow, in particular, the simulation of the electron energy loss down to thermalization $(0.025 \mathrm{eV})$. In the simulations presented here, however, only inelastic scattering (ionization and excitation) and elastic scattering were used and electrons were transported down to the last excitation shell of the liquid water molecule $(\sim 8 \mathrm{eV})$ that was used as the target material.

In the interest of saving calculation time, the nanometric detailed electron transport was only done inside the cell nuclei. Outside, and in order to achieve the electron equilibrium needed in the definition of the mean absorbed dose, electron interactions were simulated using the Livermore low energy process taking into account ionization, Bremsstrahlung and multiple scattering. Combining different models in the Monte Carlo transport of particles in different geometrical regions is possible since the 9.3 version of Geant4 [6].

\subsection{Target volumes studied}

In order to study the variance of the $f(z, D)$ distribution in an endothelium cell population more than 500 cell nuclei were simulated. Each of these cell nuclei was defined by an ellipsoid with half axis of 9.55, 5.5 and $1.0 \mu \mathrm{m}$ which corresponds to the mean characteristic values of the endothelium population studied in this work. The fluency (number of incident source particles for a run) used in the simulation corresponds to the correct value of the absorbed dose in a macroscopic volume $\left(\mathrm{mm}^{3}\right)$.

In a first calculation and as a first approximation, the distribution of the $f(z ; D)$ values was calculated from the energy deposited in the whole and homogeneous cell nuclei. In a second calculation, the objective was to take into account the fact that DSB can only appear on the DNA molecule (backbone region) which is not a continuous homogeneous target. This fact is very important because different radiation qualities (ex. ${ }^{137} \mathrm{Cs}$ and $20 \mathrm{keV}$ ) differ in the in their energy transfer point homogeneity at the sub-nuclear scale as it can be seen in Figure 1 and thus taking an homogeneous target or a discrete target with the same equivalent volume inside the cell nucleus changes the $f(z ; D)$ results.
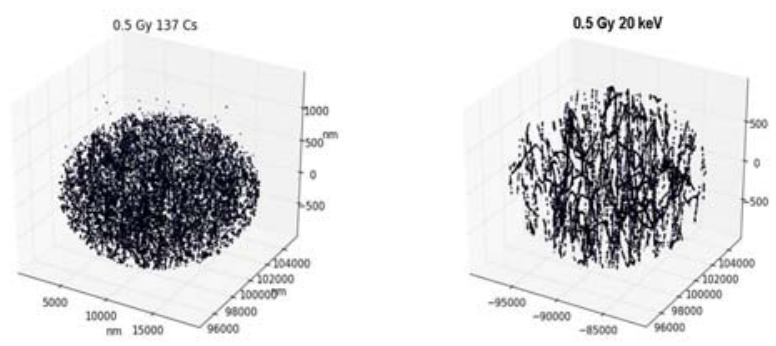

Figure 1. Two images showing the difference in the energy transfer point distribution within a typical endothelium nucleus for a ${ }^{137} \mathrm{Cs}$ (left) irradiation and a $20 \mathrm{keV}$ (right) photon irradiation for the same macroscopic absorbed dose ( 0.5 Gy). This difference can be explained from the different type of interactions for the two radiation qualities (Compton interaction for Cs and photoelectric in the case of the $20 \mathrm{keV}$ photons). The simplified model of the 'DNA target volume' proposed in this work takes into account this difference in the deposited energy distributions.

The purpose was then to simulate this corresponding sub-nuclear volume in a realistic way. Indeed, in order to compare the calculated specific energy distribution on the DNA with the biological foci distribution, it was necessary to take into account the fact that endothelium cells are irradiated in the G0/G1 phase of their cell cycle which corresponds to a DNA content almost uniformly distributed. Therefore, the sub-nuclear target volume for the calculations (corresponding to the backbone region) must be distributed almost uniformly inside the whole cell nucleus volume. To do so, a first attempt was to perform the Monte Carlo calculation within a geometrical model (M. Dos Santos and al. this conference) where the different elements of the endothelium DNA molecule are defined and structured in a realistic way (bases, backbone region, chromatin fibres, chromatin loops), in order to quantify the energy transfer points of the irradiation located in the backbone region. Unfortunately, the calculation time needed to simulate the multi-event photon irradiations was excessive and thus a simplified target model was needed. This simplified model had to reflect nevertheless, the relative position of the sensitive volumes (backbone region) in the cell nuclei to be intercepted by the 
radiation. The solution used in this work was to take as a basic volume element a small sphere of $2.85 \mathrm{~nm}$ of radius. The volume of this elementary sphere is then 97 $\mathrm{nm}^{3}$ that is equivalent to the volume occupied by the backbone region of one nucleosome $(\sim 200 \mathrm{bp})$. Knowing that the DNA of human cell nuclei is composed of around $6 \mathrm{Gbp}$, then $3 \mathrm{e}^{7}$ small spheres are needed in order to simulate the total DNA sensitive volume for DSB induction. In order to reproduce the G0/G1 phase of the cell cycle, the $3 \odot 10^{7}$ small spheres where uniformly distributed inside each cell nucleus (defined by the ellipsoid with half axis of 9.55, 5.5 and $1.0 \mu \mathrm{m})$. By doing this, the $f(z ; D)$ distribution was calculated for more than 500 targets, each target being the addition of the $3 \odot 10^{7}$ small spheres that we will call the 'DNA volume'. The $f(z ; D)$ distribution obtained in that manner, respects then the heterogeneity in the energy deposition that differs from one radiation quality to the other.

\subsection{Simulated irradiation qualities}

Two different beams were simulated: the gamma experimental spectra of an IBL-637 irradiator containing a ${ }^{137} \mathrm{Cs}$ source and a mono-energetic $20 \mathrm{keV}$ photon beam. The results of the simulations for the ${ }^{137} \mathrm{Cs}$ source at an absorbed dose of 1 Gy were compared to the $\gamma-\mathrm{H} 2 \mathrm{AX}$ foci distributions, experimentally obtained. Monte Carlo calculations were also used to study the evolution of the relative variance of the specific energy distribution of the ${ }^{137} \mathrm{Cs}$ source for different mean absorbed doses.

Finally, the influence of the radiation quality or LET was evaluated by comparing the $z$ distributions of the ${ }^{137} \mathrm{Cs}$ irradiation and a monoenergetic $20 \mathrm{keV}$ X-ray source.

\subsection{The experimental $\gamma$-H2AX foci distribution acquisition}

Radioinduced DSB in DNA can be localized within the cell nuclei by a fluorescence labeling of $\gamma$-H2AX around the damages [1]. Nevertheless, DSB are also produced during the cell cycle, mainly during the cell duplication. In order to obtain a good evaluation on the number of DSB produced by irradiation it is then necessary to control the cell cycle and irradiate the cells in the cycle phase were the number of non-radioinduced DSB is minimum, the G0/G1 phase. Endothelium cells (umbilical vein human cells) were grown, irradiated and visualized in the same microscope slide, preserving the mean geometrical parameters of the cell nuclei between the irradiation and the image analysis.

Confluent endothelium cells were then exposed in an IBL-637 irradiator to a ${ }^{137} \mathrm{Cs}$ source for a macroscopic absorbed dose of 1 Gy delivered in around 1 minute. An analysis of the cinematic of appearance of the $\gamma$-H2AX foci signal was done showing a maximum in the number of foci 30 minutes after irradiation.

The detection of the number of foci per cell was carried out using a combination of a high speed microscopy platform and an automated image analysis based on the LabVIEW technique. This allows a good evaluation of the number, position and shape of the foci on more than 10000 irradiated cells. Thus, a meaningful statistic determination of the variance on the foci distribution could be obtained.

\section{Results and discussion}

\subsection{Differences in the specific energy distribution with the target geometry}

We report here the differences obtained in the specific energy distributions depending on the target geometry. The $f(z ; D)$ calculated from all the energy transfer points contained in the cell nuclei is centered in the mean absorbed dose (0.98 Gy) and shows a variance of 0.007 ( $\mathrm{SD}=0.08 \mathrm{~Gy})$. Nevertheless, as explained in section 2.2, the target of interest in this work is the DNA volume represented by the $3 \odot 10^{7}$ small spheres uniformly distributed within the cell nuclei volume. In this case, the fit of the obtained results show a larger distribution centered in 0.90 Gy with a variance of 0.023 (SD $=0.15$ Gy) (Figure 2). Thus, this variance represents better the real heterogeneity in the absorbed energy by the DNA targets in the cell irradiation which is the one that should be compared to the experimental biological data.

\subsection{Comparison between the calculated specific energy distribution and the biological data}

In Figures 2 and 3 the Monte Carlo simulated specific energy distribution on the DNA volume and the observed $\gamma$-H2AX foci distribution after 30 minutes of irradiation are shown respectively. In both distributions, the obtained data have been fitted and their variance calculated. The relative variance ( $\sigma /$ mean value) of the foci distribution is larger than that of the $z$ distribution: $30 \%$ for the foci distribution and $17 \%$ for the $\mathrm{Z}$ distribution.

D 1 Gy- 137 Cs source. $Z$ distribution in DNA volumes

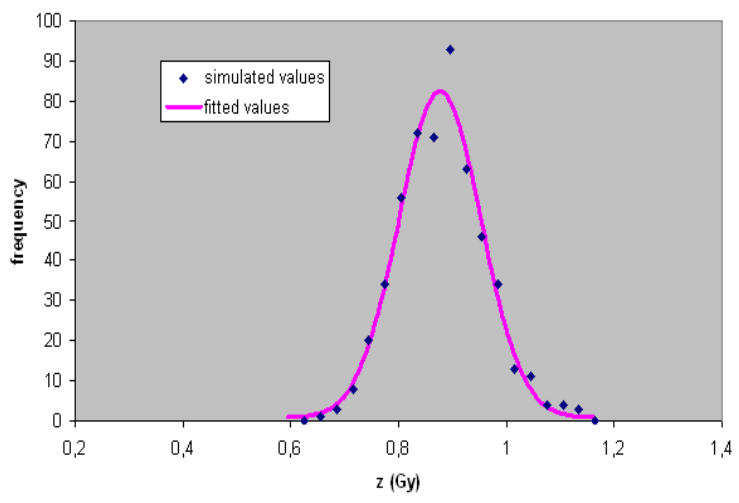

Figure 2. Specific energy distribution $f(z ; D)$ obtained by Monte Carlo simulation in an equivalent DNA volume (see $\S 2.2$ ) for a $1 \mathrm{~Gy}{ }^{137} \mathrm{Cs}$ irradiation. Calculated values (symbols) are fitted $(\mathrm{SD}=0.15 \mathrm{~Gy})$. 


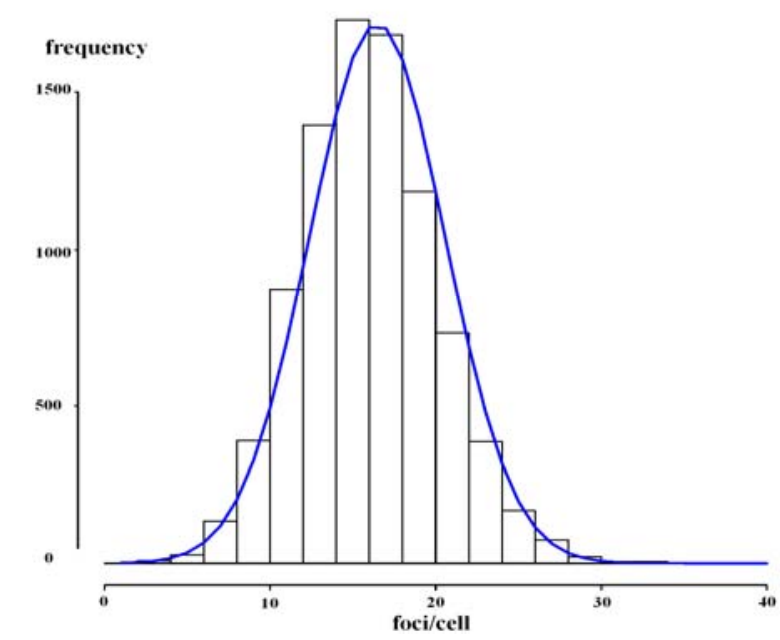

Figure 3. $\gamma-\mathrm{H} 2 \mathrm{AX}$ foci distribution obtained by an immunofluorescence technique on more than 10000 endothelium nuclei after 30 minutes of irradiation. The irradiation quality is the same that has been simulated in the results of figure 1 .

A foci distribution larger than the specific energy distribution $f(z ; D)$ assessed by calculations was expected as the image obtained 30 minutes after irradiation reflects all the processes that take place between the irradiation and the biological repairing process. Indeed, after the energy transfer between the ionizing particle and the target, known as physical stage $\left(10^{-18}-10^{-14} \mathrm{~s}\right)$, molecules around the DNA that have been ionized or excited become radicals $\left(10^{-14}-10^{-12} \mathrm{~s}\right)$. These radicals, together with the solvated electrons, diffuse and chemically react during the chemical stage $\left(10^{-12}-10^{-5} \mathrm{~s}\right)$. Interactions with the DNA molecule are then at the origin of indirect damages (as distinct from direct damages that are created during the physical stage from energy transfer points located on the backbone region). The $\gamma-\mathrm{H} 2 \mathrm{AX}$ foci result from both direct and indirect effects and thus the comparison with the specific energy $(z)$ distribution that corresponds only to the dispersion values on the physical stage can not be done directly.

In Figure 4 the comparison is done by a quantile plot of both distributions in order to check if the two data sets (the experimental foci data and the calculated $\mathrm{z}$ values) come from populations with a common distribution. As it can be seen, the correlation is almost linear showing that their distributions are comparable. This correlation suggest that, even if chemical and biological processes take place in the cell nucleus after irradiation, the differences in the initial energy deposited within the real target of the damages, namely the DNA volume, can still be seen in the number of DSB represented by the foci.

\subsection{Evolution of the specific energy distribution with the absorbed dose}

In Figure 5 the specific energy distributions for three different absorbed doses corresponding to $2 / 3 \mathrm{D}, \mathrm{D}$ and
Quantil representation

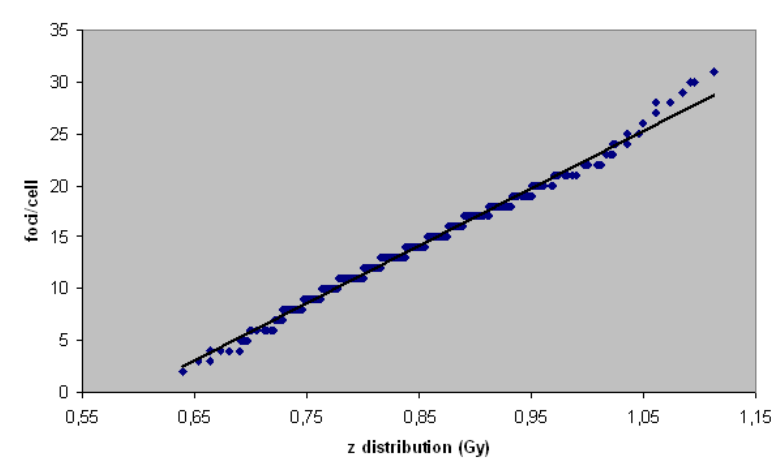

Figure 4. Quantil representation of the biological experimental data versus the calculated $\mathrm{z}$ values.

$2 \mathrm{D}$ is shown (D 1Gy). From these results it can be observed that, even if the total variance increases with the absorbed dose, the relative variance (divided by D) decreases. The results obtained for the ${ }^{137} \mathrm{Cs}$ are $19.5 \%$, $17 \%$ and $12.6 \%$ respectively.

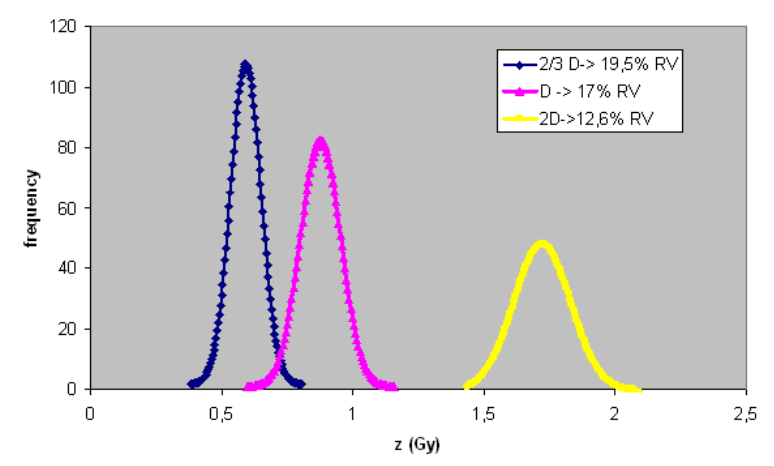

Figure 5. Evolution of the specific energy distribution with the absorbed dose for the same irradiation quality $\left({ }^{137} \mathrm{Cs}\right)$. The relative variance decreases with the absorbed dose.

\subsection{Evolution of the specific energy distribution with the radiation quality, preliminary results}

As the dispersion in the specific energy distribution is related to the heterogeneity in the energy deposition and the size and geometry of the target, it is expected to change with the radiation quality or LET. It is indeed observed in the results obtained the simulations with a $20 \mathrm{keV}$ monoenergetic photon source. In this case, the main interaction between the photons and the water target presents a greater heterogeneity in the energy deposition in the DNA target (taken as the $3 \circ 10^{7}$ small spheres uniformly distributed within the cell nuclei volume). The resulting specific energy distribution shows a value of the relative variance at 1 Gy of $23 \%$ to be compared with $16 \%$ for the ${ }^{137} \mathrm{Cs}$ spectrum that was obtained at the same macroscopic absorbed dose. These results must now be confirmed by the corresponding experimental foci data planned by the radiobiologist group. 


\section{Conclusion and perspectives}

Monte Carlo calculations using the Geant4-DNA processes of the Geant 4 code allowed us to simulate the dispersion on the specific energy imparted to nanometric targets representing the size and shape of the DNA content of an endothelium population. These results have been compared for ${ }^{137} \mathrm{Cs}$ to the experimental $\gamma-\mathrm{H} 2 \mathrm{AX}$ foci distribution detected after 30 minutes of irradiation that is related to the number of DSB created by the exposure. The comparison indicates that both data set come from a common distribution and that, even if their relative variance is not the same, the variance of the energy deposited in the target is conserved in the foci image. These results let us expect a direct link between the energy deposition structure and the quantification of these early radioinduced damages.

Moreover, Monte Carlo calculations have been used to evaluate the evolution of the $z$ variance with the absorbed dose and the radiation quality. This evolution must now be compared to ongoing experimental results on the corresponding foci distributions. If these results are confirmed, they could become a step forward to a predictive model on the risk assessment for low doses exposure.

\section{References}

[1] S. Roch-Lefèvre, T. Mandina, P. Voisin, G. Gruel, J.E. Mesa, M. Valente, P. Bonnesoeur, O. Garcia, Pa. Voisin and L. Roy, Quantification of $\gamma-\mathrm{H} 2 \mathrm{AX}$ foci in human lymphocytes: A method for biological dosimetry after ionizing radiation exposure, Rad. Res. 174, 2 (2010), pp. 185-194.

[2] M. Löbrich, A. Shibata, A. Beucher, A. Fisher, M. Ensminger, A.A. Goodarzi, O. Barton and P.A. Jeggo, $\gamma \mathrm{H} 2 \mathrm{AX}$ foci analysis for monitoring DNA double strand break repair, Cell Cycle 9:4 (2010), pp. 662-669.

[3] E.L. Leatherbarrow, J.V. Harper, F.A. Cucinotta and P. O'Neill, Induction and quantification of $\gamma-\mathrm{H} 2 \mathrm{AX}$ foci following low and high LET-irradiation, Int. J. Radiat. Biol. Vol.82, No.2 (2006), pp. 111-118.

[4] S. Agostinelli, J. Allison, K. Amako et al., GEANT4-a simulation toolkit, Nucl. Instrum. Methods. Phys. Res. A. 506 (2003), pp. 250-303.

[5] C. Villagrasa, Z. Francis and S. Incerti, Physical models implemented in the GEANT4-DNA extension of the GEANT4 toolkit for calculating initial radiation damage at the molecular level, Radiat. Prot. Dosim. 143 (2011), pp. 214-218.

[6] V. Ivanchenko, J. Apostolakis, A. Bagulya et al., Recent improvements in Geant4 electromagnetic physics models and interfaces, Progress in Nucl. Sci. and Tech. Vol.2 (2011), pp. 898-903. 